\title{
Commentary on "A Case of Erdheim-Chester Disease with Asymptomatic Renal Involvement"
}

Gioacchino Li Cavoli, MD

Division of Nephrology and Dialysis, Civic and Di Cristina Hospital, Palermo, Italy

We read with interest the article written by Dr. Lee et al. [1] on renal involvement in Erdheim-Chester disease (ECD). We agree with the authors on the long asymptomatic period of obstructive nephropathy that occurs in ECD. In our experience, a 60-year-old female, suffering from diabetes mellitus, who was treated with metformin, was admitted for fever, abdominal pain, diarrhoea, and acute renal failure (creatinine, $19 \mathrm{mg} / \mathrm{dL}$ ). For two years, she had suffered from ECD, diagnosed in another medical ward. She had undergone interferon therapy with stability of her clinical status. Since the diagnosis, she had bilateral hydronephrosis, however, she had never suffered from impaired renal function. At admission, laboratory examinations showed: leukocytes, 21,500/ $\mu \mathrm{L}$; platelet, $54,000 / \mu \mathrm{L} ; \mathrm{Hb}, 11 \mathrm{~g} / \mathrm{dL} ; \mathrm{pH}, 7.05 ; \mathrm{HCO}^{3-}, 9 \mathrm{mEq} / \mathrm{L}$; serum lactates, $6 \mathrm{mEq} / \mathrm{L}$. Findings on other routine laboratory examinations were within the normal range. Renal ultrasound showed a bilateral hydronephrosis, more to the right side. A percutaneous nephrostomy was placed to the right side; the patient received antibiotic and saline solution therapy with progressive improvement of her clinical status (remission of fever, recovery of diuresis; normalization of renal function and acid-base balance); blood and urine cultures were negative for bacterial growth. We diagnosed intestinal discomfort and lactic acidosis metformin associated with acute renal failure secondary to dehydration. Upon the complete normalization of renal function (creatinine, 1.16 $\mathrm{mg} / \mathrm{dL}$ ), it was possible to perform a computed tomography scan with contrast, which showed a slight hydronephrosis on the right side (Fig. 1) and a marked hydronephrosis on the left side (Fig. 2). We decided to remove the percutaneous nephrostomy, and, as in the case reported by Dr. Lee et al. [1], we counseled a wait-and-see policy in the outpatient clinic. We counseled ureteral stenting in cases of hydronephrosis or deterioration of renal function.

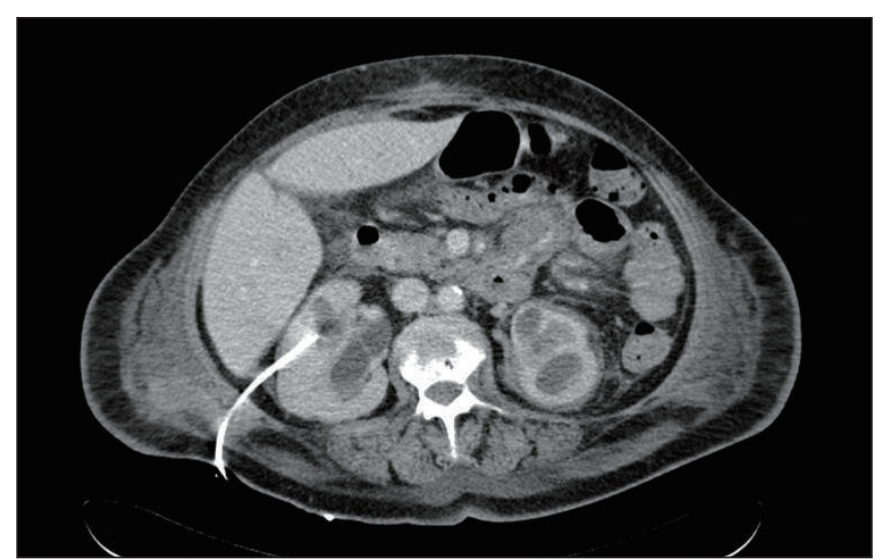

Fig. 1. Computed tomography abdomen scan: slight hydronephrosis of right kidney with percutaneous nephrostomy.

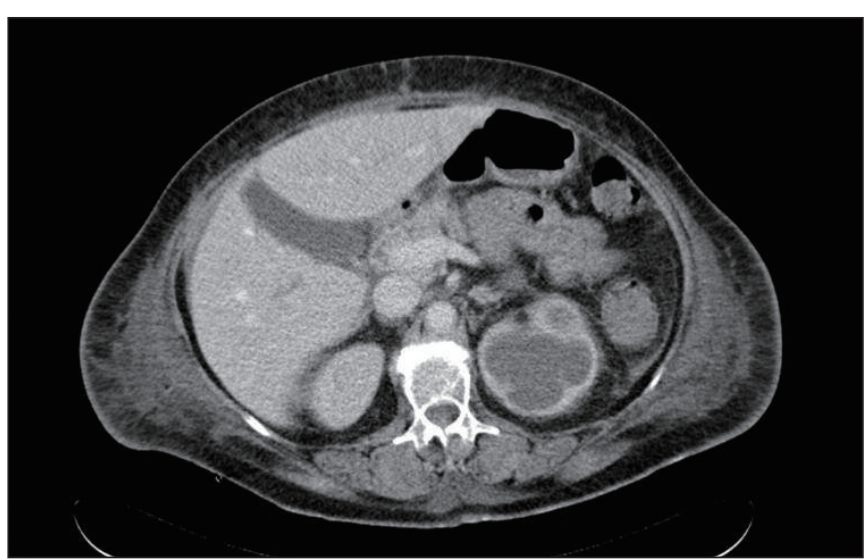

Fig. 2. Computed tomography abdomed scan: marked hydronephrosis of left kidney.

\section{Reference}

1. Lee HJ, Lee KY, Shin DY, Lee YG, Choi SY, Moon KC, et al. A case of Erdheim-Chester disease with asymptomatic renal involvement. Cancer Res Treat. 2012;44:146-50. 